\title{
Measurements of lip pressure exerted on a cigarette during normal smoking
}

\author{
ROGER D. KAMM \\ Massachusetts Institute of Technology, Cambridge, Massachusetts
}

\begin{abstract}
Measurements were made of the pressure exerted by human lips on the filter of a cigarette during normal smoking conditions using a small, fluid-filled bulb attached to a low-compliance pressure transducer. In two series of measurements, the mean lip pressure and standard error were 34.2 $( \pm 2.6)$ and $35.8( \pm 3.1)$ torr respectively. These pressures compared favorably with those applied by the Cambridge holder (used in the standard FTC testing procedure), but were much lower than the pressures exerted by the Filtrona and Borgwaldt holders, both of which are constructed from a rigid cylindrical housing with a segment of latex tube mounted inside. Pressures measured by these two holders were in close agreement with the pressures predicted by a nonlinear theoretical model for the distention of latex rubber tubes.
\end{abstract}

Although factors relating to smoking behavior have been subjects of various studies, these factors have not included interactions between the smoker's lips and the cigarette filter. This probably is due to the assumption that, for most brands of cigarette, the lip-filter interaction has little effect on the tar and nicotine levels the smoker receives. The lips actually may play a significant role in determining the levels of tar and nicotine in the smoke of all cigarettes that are ventilated. If the lips impair the normal flow of ventilation air, the delivery of tar and nicotine could be considerably increased from the levels indicated by the accepted testing procedures. Obviously, if either the lips or the fingers of the smoker cover the ventilation holes, then the smoker will, in effect, be smoking a much stronger cigarette. Finger blockage has, in fact, been observed in as many as $40 \%$ of all ventilatedcigarette smokers, according to a recent study (Kozlowski, Frecker, Khouw, \& Pope, 1980).

Recently a new type of filter has been introduced that may be susceptible to a somewhat different type of smoker "sabotage." This filter also has ventilation perforations at approximately the same location on the filter as other conventional filters, but these perforations directly communicate with only four small grooves that run beneath the filter paper to the end of the filter. If these grooves were to collapse due to pressures exerted by the lips during smoking, then once again the smoker would receive more smoke than would a testing machine. This statement is based on the assumption that the holder used during testing does not compromise the flow of ventilation air in any way due, for example, to pressures that are unrealistically high. Clearly, the pressures exerted by human lips, compared with pressures applied to the filter

Please address all correspondence to: R. D. Kamm, Massachusetts Institute of Technology, 77 Massachusetts Avenue, Room 3-260, Cambridge, MA 02139. by the various filter holders used in cigarette testing, are important.

I have conducted a series of tests under conditions simulating normal smoking in which the contact stress acting between the smoker's lips and the cigarette filter was measured. The pressures exerted by three different types of cigarette holder have also been measured and compared with the measurements of lip pressure. These measurements are presented here and their import for the ventilation reaching the smoker are discussed.

Because measurements of a smoker's lip pressure have not been reported in the past, a new measurement technique was needed. This technique draws upon methods that have been used to measure contact stress in other situations, but differs in the size of the sensing element and the sensitivity of the method.

Various methods have been employed in the past to measure the contact stress acting between two solid surfaces. These methods generally measure the force applied to a known surface area by means of the deflection of a diaphragm or the change in electrical properties of a piezoelectric or piezoresistive device. Although these methods are reliable and accurate in most applications, they fail in this research either because they lack the necessary sensitivity or they cannot be sufficiently miniaturized to ensure that the act of measurement will not significantly influence the pressures we wish to determine. To ensure reliable measurement of lip pressure, a device is required which is small compared to the cigarette diameter and which conforms to the smoker's lips when contact is made. The device must be sensitive and capable of accurately measuring pressures down to 10 or 20 torr (1 torr $=1 \mathrm{~mm} \mathrm{Hg}$ ).

Similar measurements have been made inside the mouth to determine the pressures exerted between the lips and the teeth or the tongue and teeth. Intraoral pressures have been measured both by a technique similar to that employed in these lip-pressure/cigarette tests, and by using 
a sensitive strain gauge mounted on the tooth surface or in a small plastic housing. In such tests, pressures in the range of 20-150 torr have been observed in normal activities such as speaking and swallowing (Proffit, 1975).

\section{METHOD}

\section{Measurement Apparatus}

In order to satisfy the stringent requirements stated above, a miniature pressure-sensing element was developed. This miniature probe consists of a thin-walled latex rubber bulb, nearly spherical in shape (diam = $0.15 \mathrm{~cm}$ ), with a narrow throat to allow attachment to a segment of hypodermic needle tubing (length $=1 \mathrm{~cm}$, i.d. $=0.01 \mathrm{~cm}$ ). The needle tubing is connected to a Teflon catheter (length $=50 \mathrm{~cm}$, i.d. $=0.05 \mathrm{~cm}$ ) that leads to a low-compliance pressure transducer. The entire system (bulb to transducer) is filled with water to eliminate the compliance due to fluid compressiblity and therefore maximize the usable pressure range of the device. The bulb was mounted on the cigarette filter in one of two ways. In one set of experiments (Series I), the bulb was attached directly to a small exposed segment of the cellulose filter material at a distance of $7 \mathrm{~mm}$ from the tip of the filter using a fast-acting contact adhesive. In the other set (Series II), the bulb was first mounted onto a 1-cm-long segment of thin-walled latex tube (nominal i.d. $=0.79 \mathrm{~cm}$ ) which could be slipped over the end of the filter to the desired position. The latter modification was adopted merely to facilitate mounting of the probe.

The assembled apparatus is shown in Figure 1. When mounted in the fashion shown, a smoker's lips or filter holder press on the sensing probe causing the internal fluid pressure to increase. This is immediately sensed by the pressure tansducer and can be displayed on an oscilloscope screen.

Due to the properties of the bulb and to the distribution of stresses on the bulb, the recorded pressure $\left(\mathrm{P}_{\text {rec }}\right)$ differs in general from the applied pressure $\left(\mathrm{P}_{\mathrm{app}}\right)$. As an illustration, consider a general functional relationship between the volume of the sensing bulb $\left(\mathrm{V}_{\mathrm{bulb}}\right)$, the pressure difference acting across the bulb $\left(\mathrm{P}_{\mathrm{app}}-\mathrm{P}_{\mathrm{rec}}\right)$, and the distribution of pressure acting on the external surface of the bulb $\left[D\left(r_{s}\right)\right.$, where $r_{s}$ denotes position on the bulb surface]:

$$
\mathrm{V}_{\text {bulb }}=\mathrm{V}_{\text {bulb }}\left[\mathrm{P}_{\mathrm{app}}-\mathrm{P}_{\mathrm{rec}}, \mathrm{D}\left(\mathrm{r}_{\mathrm{s}}\right)\right] .
$$

Because the volume of incompressible fluid within the measurement system is constant, changes in bulb volume $\left(\Delta V_{\text {bulb }}\right)$ and changes in transducer and tubing volume $\left(\Delta V_{\text {trans }}\right)$ must be of equal magnitude but opposite sign. Therefore, we can write:

$$
\mathrm{V}_{\text {bulb }}=\mathrm{V}_{\text {bulb,i }}+\Delta \mathrm{V}_{\text {bulb }}=\mathrm{V}_{\text {bulb,i }}-\Delta \mathrm{V}_{\text {trans }}
$$

Note that $\Delta \mathrm{V}_{\text {trans }}$ is a function only of the internal pressure and therefore can be stated:

$$
\Delta \mathrm{V}_{\text {trans }}=\Delta \mathrm{V}_{\text {trans }}\left(\mathbf{P}_{\text {rec }}\right) .
$$

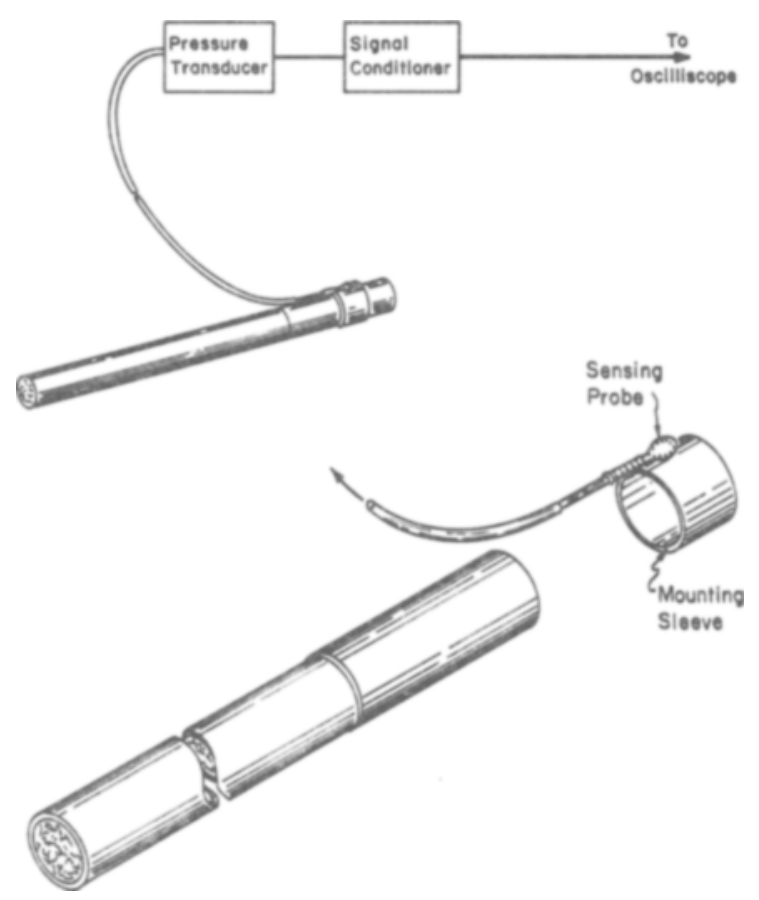

Figure 1. Measurement apparatus and associated electronics.

After combining Equations 1-3, the following functional relationship can be deduced:

$$
P_{\text {app }}=h\left[P_{\text {rec }}, D\left(r_{s}\right)\right] .
$$

Therefore, to the extent that the distribution of external pressure is either relatively constant or unimportant, $P_{\text {rec }}$ will be directly related to $P_{a p p}$.

Because the exact form of the function $h\left[P_{r e c}, D\left(r_{s}\right)\right]$ is not known, a calibration apparatus was developed and used with each individual test. The device (Figure 2) was designed to mimic as closely as possible the distribution of pressure produced by the lips, thus maintaining $D\left(r_{s}\right)$ roughly constant. The calibration device consists of two rigid Plexiglas plates, each machined to accept a cigarette, and a 10-cm length of thin-walled latex rubber tubing. When assembled, the latex tube is positioned directly over the sensing bulb, and pressure applied inside the tube is directly exerted on the sensing bulb. We can assume that the pressure difference across the wall of the latex sleeve is small due to the large local radius of curvature of the latex relative to that of the sensing probe. A calibration curve was obtained by inflating the latex tube to approximately 5 or 6 levels of pressure within the range of interest. The relationship was plotted as $E_{\text {rec }}$ versus $P_{a p p}$ ( $E_{\text {rec }}$ is the output voltage of the transducer associated with $\left.P_{\text {app}}\right)$ as in the sample curve given in Figure 3.

\section{Protocol for Lip Pressure Measurements}

In preparation for an experiment, the sensing probe was mounted onto a cigarette in one of the two ways described 
above. The smoking panelist was then brought into the room and was seated at a table on which the instrumented cigarette had been placed. In all cases the cigarette was the usual brand of the panelist. Each panelist was asked to smoke in a normal fashion and at a normal pace, taking care to position the small sensing probe squarely against his/her lip. For each test, we obtained approximately 10 pressure measurements: 5 with the bulb positioned against the lower lip and 5 with the bulb positioned against the upper lip, the order of which was random. The pressure excursions during each puff were monitored using an oscilloscope, and the maximum deflection for each puff was recorded.

During the course of measurement, it was occasionally necessary to guide the panelist with regard to bulb position. Observing the pressure on the oscilloscope, we could see immediately when the panelist's lip had missed the bulb. All cases in which the bulb was missed were ignored.

Each cigarette was calibrated using the procedure described above either prior to or following the test. In several cases the calibration was performed both before and after the lip pressure measurements to ensure consistency of the calibration relationship.
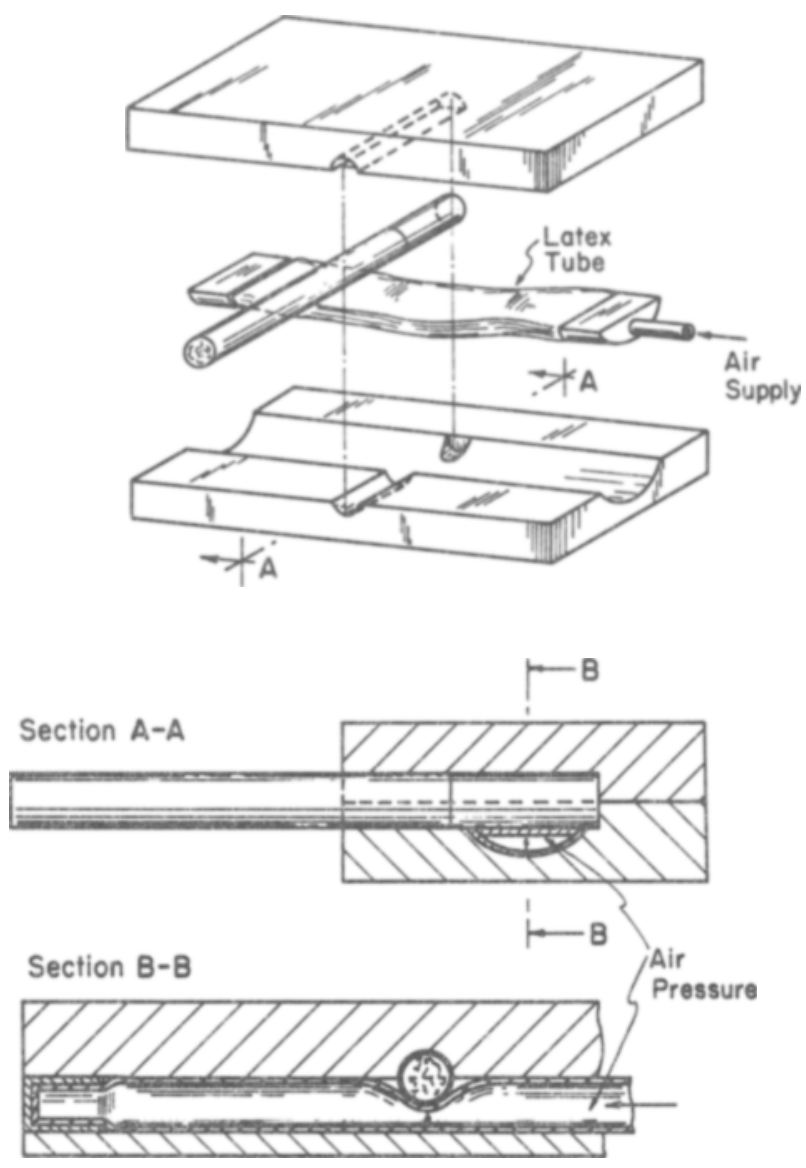

Figure 2. Top: Assembly of calibration device. Bottom: Section views of calibration device when assembled.

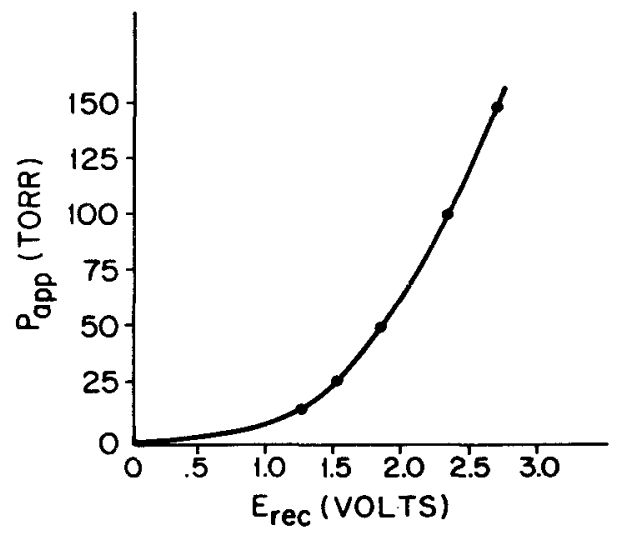

Figure 3. Sample calibration curve.

In the two series of tests, usable data were obtained on 12 and 18 subjects, respectively. The first set of subjects were regular smokers of Barclay cigarettes; the second set regularly smoked Rich Lights. Three sets of data were not used due to one of two problems: either the bulb ruptured during the test, or the Teflon catheter came too close to the burning ash and melted. The results are summarized in Table 1.

\section{Filter-Holder Measurements}

The measurements of pressure exerted on the three filter holders were conducted with the same instrumented cigarette, calibrated in the manner described above. In each case, several pressures were recorded at the position of peak pressure within the holder and are summarized in Table 2. The three holders tested are: (1) Cambridge holder, the Federal Trade Commission standard, is made of a rigid plastic holder on which a latex rubber sheet can be mounted. The latex sheet used in this test was the industry standard, 0.20-0.23 mm thick with a 3-mm-diam hole. When stretched onto the holder, the hole diameter increases to $3.9 \mathrm{~mm}$. (2) Borgwaldt holder is made from a rigid, cylindrical housing with a latex tube slipped inside and stretched over the two ends. The standard latex sleeve used in our tests had o.d. = $7 \mathrm{~mm}$ and wall thickness $=0.36-0.43 \mathrm{~mm}$. (3) Filtrona holder consists of a cylindrical housing with a latex sleeve inside, mounted on the two ends. It differs form the Borgwaldt mainly in terms of the tube dimensions (o.d. = $6 \mathrm{~mm}$; wall thickness $=0.38 \mathrm{~mm}$ ). The Borgwaldt and Filtrona represent a holder design sometimes used for cigarette testing in Europe.

\section{RESULTS}

\section{Lip Pressure Measurements}

Our objective was to measure the pressures exerted by human lips during normal cigarette smoking and to compare these pressures to those produced by various types of cigarette holders used for testing purposes. The values of lip pressure presented in Table 1 fall within a wide 
Table 1

Data Summary: Lip Pressure Measurements

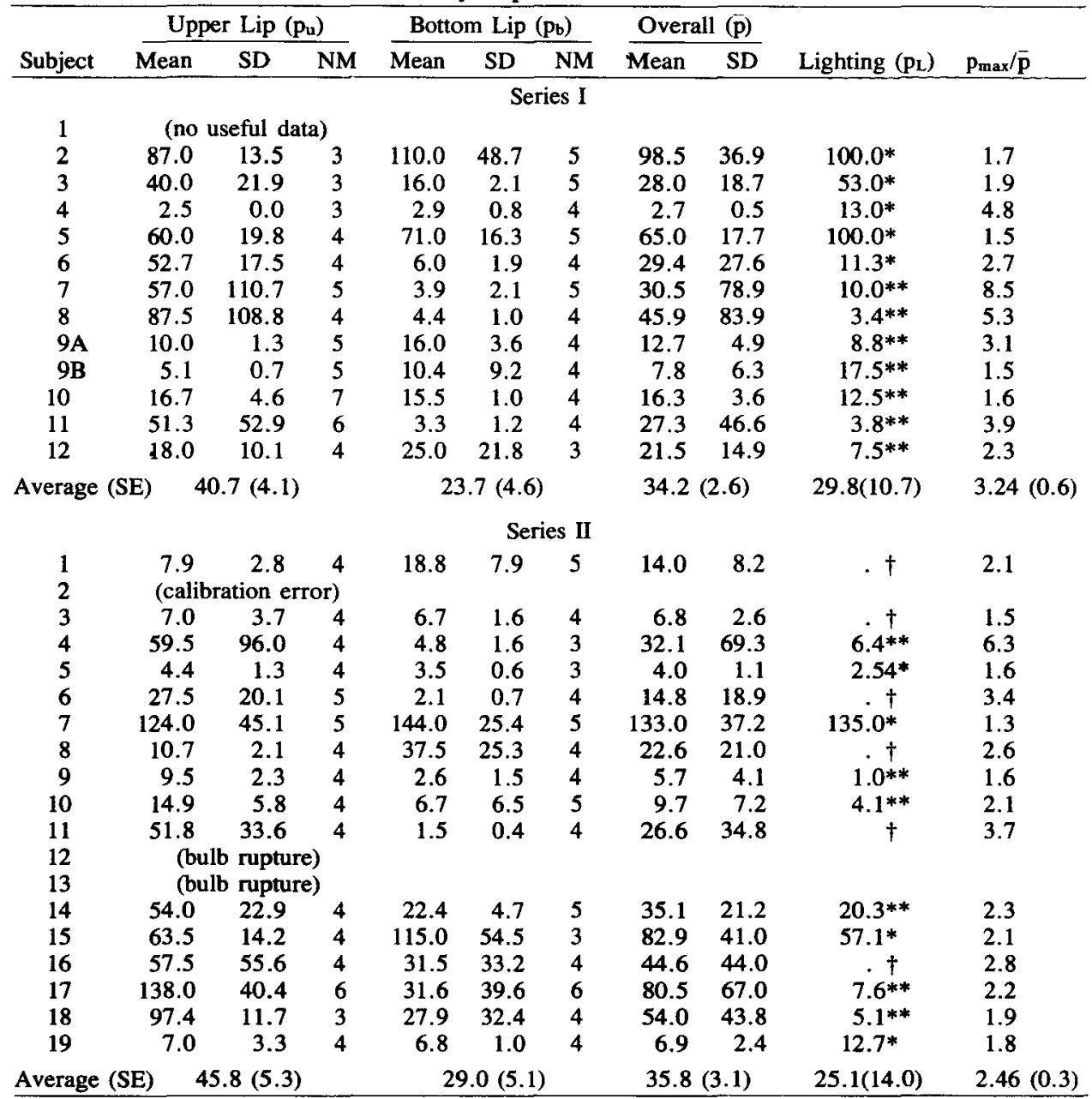

Note-All pressures given in torr. $N M=$ number of measurements. ${ }^{*}$ Lighting with bulb against upper lip. **Lighting with bulb on lower lip. $\quad+B u l b$ not against lip.

range with a mean value of 34 torr. This pressure was lower than that of the filter holders tested, but was closest to the pressures produced by the Cambridge holder.

The scatter in the data reported in Table 1 is due to a variety of factors. First, there are obvious differences in individuals' jaw structures, muscle tone, and smoking behavior. Even the same individual exhibited a wide degree of variability from one draw to the next. Thus, it is useful to look at a comparison of the overall mean pressure and the maximum pressure for each subject (Table 1). Typically, the maximum pressure was about twice as high as the overall mean. Five subjects, however, exhibited values of $\mathrm{p}_{\max }$ greater than four times as high as the mean. We suspect that this was due to the panelists' teeth coming into contact with the sensing bulb. Another source of variability was the positioning of the bulb beneath the lips. The contact stress acting between the filter and lips was distributed nonuniformly both along the axis of the cigarette and around the circumference. By instructing the panelist to place the bulb squarely against either the up- per or lower lip, we attempted to obtain a measurement near the maximal point of this distribution. Due largely to the considerable variability of insertion depth from smoker to smoker, however, bulb contact was often difficult to accomplish. Failure of lip/bulb contact was most vividly demonstrated by the total absence of signal on the oscilloscope when the panelist's lips missed the probe altogether. Therefore, the measurements must be viewed as approximate peak pressures and, based on the variability of our measurements, the actual peak pressure during any particular puff may typically be two to three times as large as the mean value given in Table 1 .

Table 2

Filter-Holder Pressures

\begin{tabular}{lcc}
\hline Filter Holder & Peak Pressure & Measurements \\
\hline Cambridge & 47 & 2 \\
Borgwaldt & 118 & 3 \\
Filtrona & 500 & 15 \\
\hline
\end{tabular}

Note-All pressures given in torr. 
The two brands of cigarette used in these tests apparently had little or no effect on lip pressure. One might speculate, however, that cigarettes with a particularly hard draw might lead to higher pressure associated with the greater effort required. Similarly, cigarettes with little taste might also lead to greater pressure as the smoker draws in more volume to satisfy his or her need.

The pressures we observed are in approximate agreement with measurements of pressure in a region lingual to the maxillary molars during the act of speaking, for example. Using measurement probes mounted in plastic, Proffit (1975) found contact stresses to lie in the range of 10-60 torr for teenage Caucasian subjects.

All measurements reported here pertain to the maximum pressure excursion for each individual draw on the cigarette. The peak is recorded during a pressure history that typically has the appearance of the trace shown in Figure 4. Although deflections in this trace are not linearly related to lip pressure, we can still draw inferences from its general shape. Characteristically, the signal would deflect rapidly and reach an early maximum as the smoker placed the cigarette into his or her mouth and began to draw. Sometimes we observed a single plateau during this rising phase corresponding to a short pause between cigarette insertion and the onset of draw. The pressure would fall more or less rapidly as the draw progressed, level off at the end of the draw, and then fall to zero as the cigarette was removed from the mouth. From traces like this, we are able to conclude that the pressure maximum is of very short duration and that the time-mean pressure during the draw is on the order of half the peak value.

\section{Filter Holders}

The three filter holders were found to exhibit grossly different characteristics both in terms of the values of peak contact stress and in the distribution of that stress upon the filter. Of the three filter holders tested, only the Cambridge holder came close to exerting pressures comparable to those produced by the lips. The pressures exerted by the Borgwaldt and Filtrona holders were, respectively, about 4 and 15 times larger than lip pressure. The differences between these two holders can be attributed entirely to the proportion and dimensions of the tubing, as demon-

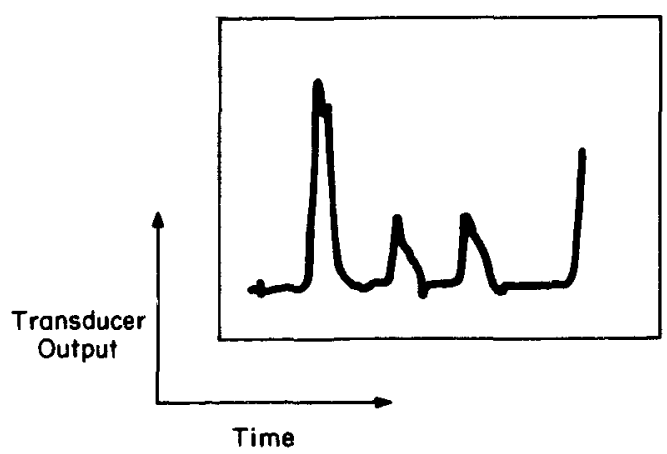

Figure 4. Transducer output during three consecutive puffs.

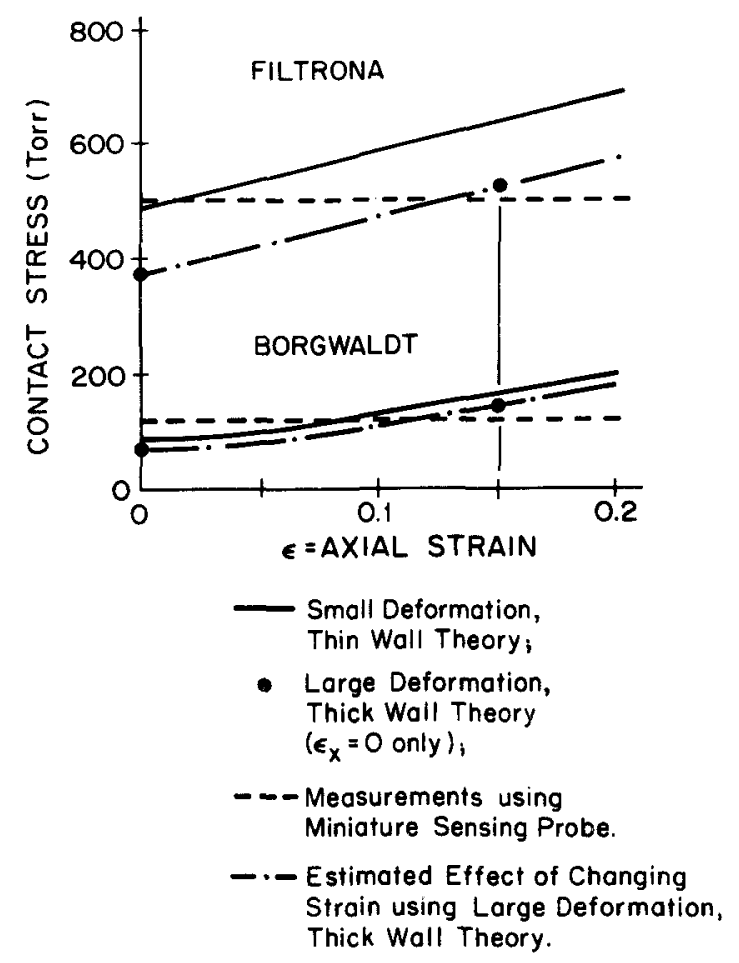

Figure 5. Predicted and measured contact stress with the Filtrona and Borgwaldt holders.

strated in the Appendix. The pressures are not only lower with the Cambridge holder, but also act over a smaller surface area as compared with the Borgwaldt or Filtrona holders. The region of contact in all cases is symmetric around the filter circumference but ranges in length from about $3 \mathrm{~mm}$ in the Cambridge to about $10 \mathrm{~mm}$ in the Borgwaldt and Filtrona.

These measurements of filter-holder pressure provide us with an additional check on the validity of our measurement technique. By performing the analysis described in the Appendix, it is possible to predict the approximate pressure exerted by either of the tube-in-cylinder holders (Borgwaldt or Filtrona). Accounting for an axial strain of about $15 \%$ imposed during the mounting procedure and for the effects of finite deformation and wall thickness, we predicted pressures of around 520 torr for the Filtrona holder and 150 torr for the Borgwaldt holder (see Figure 5). These pressures are in close agreement with the measurements reported in Table 2, suggesting that our measurements are, indeed, correct and accurate.

\section{SUMMARY}

Using a pressure probe developed specifically for the measurement of lip pressure and calibrated using a device designed to simulate the action of human lips, the pressures exerted by the lips of smokers and by various types of cigarette holder have been measured. The contact stress exerted between the lips of a smoker and the cigarette filter 
were comparable to those exerted within the mouth as measured by other techniques, but were generally lower than those produced by the various types of cigarette filter holder used for testing purposes. Only the Cambridge holder (currently recommended by the FTC) produces pressures that are similar to lip pressures.

\section{REFERENCES}

Kozlowski, L. T., Frecker, R. C., Khouw, D., \& Pope, M. H. (1980). Misuse of "less hazardous" cigarettes and its detection: Holeblocking of ventilation filters. American Journal of Public Health, 70, 1202-1203.

Proffit, W. R. (1975). Muscle pressures and tooth position: North American Whites and Australian Aborigines. Angle Orthodontist, 45, 1-11.

Taylor, L. A., \& Gerrard, J. H. (1977). Pressure-radius relationships for elastic tubes and their application to arteries. Part I: Theoretical relationships. Medical and Biological Engineering and Computing, 15, 11-17.

Timoshenko, S., \& Woinowsky-KRIEGER, S. (1969). Theory of plates and shells. Engineering Society Monograph. New York: McGraw Hill.

\section{APPENDIX \\ Prediction of Filter-Holder Pressures}

The measurement of filter-holder pressure proved to be useful, not only for comparison to lip pressure, but also as an independent means of calibration. Because the geometry of the two latex tube holders, Filtrona and Borgwaldt, is symmetric and relatively simple, the pressure they exert on a filter of known diameter can be prediced with reasonable accuracy.

The elastic tubes used in these two holders are subjected to both axial and circumferential strain denoted by $\epsilon_{\mathrm{x}}$ and $\epsilon_{\theta}$, respectively. If the tube wall is assumed to behave as a homogeneous and isotropic thin-walled membrane, then these strains are related to the circumferential tension through (Timoshenko \& Woinowsky-Krieger, 1969):

$$
\mathrm{T}=\operatorname{Eh}\left(\epsilon_{\theta}+\sigma \epsilon_{\mathrm{x}}\right) /\left(1-\sigma^{2}\right),
$$

where $\mathrm{E}$ is the Young's modulus of the material, $\sigma$ is Poisson's ratio and $h$ is the tube wall thickness. The axial stress satisfies:

$$
\mathrm{Q}=\operatorname{Eh}\left(\epsilon_{\mathrm{x}}+\sigma \epsilon_{\theta}\right) /\left(1-\sigma^{2}\right)
$$

The pressure difference across the membrane is related to the membrane tension and local radius of curvature in two mutually perpendicular planes and is given by:

$$
\Delta \mathrm{P}=\mathbf{P}_{\mathrm{int}}-\mathbf{P}_{\mathrm{ext}}=\mathrm{T} / \mathbf{R}_{\boldsymbol{\theta}}-\mathrm{Q} / \mathbf{R}_{\mathbf{x}},
$$

where, $\mathbf{R}_{\boldsymbol{\theta}}$ and $\mathbf{R}_{\mathrm{x}}$ are the radii of curvature of the surface in a cross-sectional plane and axial plane, respectively.
When the tube is mounted but before a cigarette is inserted into the holder, $P_{\text {int }}=P_{\text {ext }}$ and

$$
\mathbf{T} / \mathbf{R}_{\boldsymbol{\theta}}=\mathbf{Q} / \mathbf{R}_{\mathbf{x}}
$$

When the cigarette is in place, $\mathbf{R}_{\mathrm{x}}=\infty$ and

$$
\mathrm{T}=\mathbf{R}_{\theta} \Delta \mathbf{P}=\operatorname{Eh}\left(\epsilon_{\theta}+\sigma \epsilon_{\mathrm{x}}\right) /\left(1-\sigma^{2}\right) .
$$

Moreover, since the volume of tube wall remains constant;

$$
h R L=h_{0} R_{o} L_{o},
$$

where $h_{o}, R_{o}$, and $L_{o}$ are the thickness, radius, and length of the unstressed tube. Changes in radius and length are related to the circumferential and axial strain according to:

$$
\begin{aligned}
\epsilon_{\theta} & =\left(\mathrm{R}-\mathrm{R}_{o}\right) / \mathrm{R}_{o} \\
\epsilon_{\mathbf{x}} & =\left(\mathrm{L}-\mathrm{L}_{o}\right) / \mathrm{L}_{\boldsymbol{o}} .
\end{aligned}
$$

These expressions can be combined with Equation A-5 to yield:

$$
h=h_{o}\left(\epsilon_{\theta}+1\right)\left(\epsilon_{x}+1\right) .
$$

Substituting into Equation A-4 we obtain:

$$
\Delta \mathrm{P}=\mathrm{Eh}_{\mathrm{o}}\left(1+\epsilon_{\mathrm{x}}\right)\left\{\left[\left(\mathrm{R}-\mathrm{R}_{\mathrm{o}}\right) / \mathrm{R}_{\mathrm{o}}\right]+\sigma \epsilon_{\mathrm{x}}\right\} /\left[\mathrm{R}\left(1-\sigma^{2}\right)\right] .
$$

Of the parameters on the right in Equation A-6, only $\epsilon_{\mathrm{x}}$ is unknown but is estimated to be 0.15 for both filter holders tested. Plotting $\Delta \mathrm{P}$ as a function of axial strain, we obtain Figure 5.

The above analysis assumes both that the wall is very thin and that the stress-strain behavior of latex rubber is linear. This is, however, only an approximation, the accuracy of which decreases with increasing strain. An analysis that relaxes both of these constraints has been performed by Taylor and Gerrard (1977) for the case of zero axial strain. Their result can be written:

$$
\Delta \mathrm{P}=\frac{2}{3} \frac{E h_{o}}{D_{o}}\left(1-\frac{R_{o}^{4}}{R^{4}}\right) \frac{\left(1+h_{o} / 2 R_{o}\right)}{\left(1+h_{o} / R_{o}\right)^{2}} .
$$

The value of $\Delta \mathrm{P}$ computed by this equation is also shown in Figure 5. If we assume, without proof, that changes in axial strain affect $\Delta \mathrm{P}$ similarly for both cases, we obtain the approximate relationships indicated by the dash-dot lines in the figure.

(Manuscript received December 28, 1984; revision accepted for publication June 5,1985 .) 